\title{
Treatment of advanced peri-implantitis with regenerative therapy: a review of the rationale, technique, and outcomes
}

\author{
Stuart J. Froum \\ Department of Periodontology and Implant Dentistry, New York University College of Dentistry, New York, NY, USA \\ Correspondence to: Stuart J. Froum, DDS. Clinical Adjunct Professor and Director of Clinical Research, Department of Periodontology and Implant \\ Dentistry, New York University College of Dentistry, New York, NY, USA. Email: dr.froum@verizon.net.
}

\begin{abstract}
This article reviews a regenerative technique for treatment of implants with Advanced Peri-implantitis (where bone loss is greater than $50 \%$ of the implant length). Also reviewed are the prevalence, classification, and rationale for treatment. Literature is presented that shows regeneration (re-osseointegration) of new bone to an implant with peri-implantitis is possible in humans. An 8-step regenerative approach which has been used to successfully treat advanced peri-implantitis is presented in detail covering case selection flap access, implant surface decontamination (SDC), biologics placed on the debrided implant surface which is then covered with a bone graft and membrane barrier. The type of barrier used depends on whether there is sufficient keratinized tissue. If the latter is not present a subepithelial soft tissue connective graft is harvested from the palate to serve as the barrier. Most articles and decision trees recommend that an implant with advanced peri-implantitis be treated by implant removal, rebuilding of the site, and attempting to place another implant. The regenerative technique described in this article was used to treat 170 consecutively treated implants (48 with advanced peri-implantitis) and resulted in a $98.8 \%$ success rate with 168/170 implants retained and healthy at a 2-10 years follow up, thus avoiding implant removal.
\end{abstract}

Keywords: Peri-implantitis treatment; advanced peri-implantitis; regeneration; surface decontamination (SDC)

Received: 14 March 2021; Accepted: 05 August 2021; Published: 30 December 2022.

doi: $10.21037 /$ fomm-21-30

View this article at: https://dx.doi.org/10.21037/fomm-21-30

\section{Background}

Peri-implantitis has been defined by the First European Workshop as an inflammatory condition surrounding an implant and resulting in bleeding on probing (BOP) and loss of supporting bone (1). The 2017 World Workshop updated the classification scheme and defined periimplantitis as a plaque associated pathologic condition occurring in the tissues around dental implants, characterized by inflammation in the peri-implant mucosa and subsequent progressive loss of supporting bone $(2,3)$. Most researchers have documented bacteria plaque (now called microbiome) on the surface of the implant as the main etiologic agent of peri-implantitis (4-8). This is the reason all methods of treatment of peri-implantitis include techniques and materials to decontaminate the implant surface as an integral part of therapy.

The purpose of the present review is to examine the prevalence, classification, rationale, and treatment of implants with advanced peri-implantitis and discuss outcomes of therapy.

\section{Prevalence}

A systematic review with meta-analysis reported the prevalence of peri-implantitis to be $22 \%$ (range, 1-47\%) (9). In two other studies, 10 years post implant placement, 50\% of dental implants showed signs of peri-implantitis $(10,11)$. The consensus report of the Sixth European Workshop in Periodontology reported an incidence of periimplantitis between $28 \%$ and $56 \%$ (12). The large range recorded in these studies is mainly based on varying study designs, 
Figure 1 Classification of advanced peri-implantitis. PD, probing depth; BOP, bleeding on probing, bone loss.

population sizes and statistic profiles (13). However, when evaluating the prevalence of peri-implantitis one of the main factors contributing to the widespread differences in findings is that different definitions of the disease were used in the studies (14). In fact, in a review on the management of peri-implant disease the authors sited 13 articles with different disease definitions which would obviously lead to different values for prevalence (15).

The same would hold true for the treatment of periimplantitis in that success and failure rates vary depending on whether the specific treatment used was on implants with early, moderate, or advanced disease. This emphasizes the importance of a disease classification to determine the effectiveness of a specific treatment and the reproducibility of those results in other groups of patients with the same degree of involvement.

\section{Classification of peri-implantitis}

A number of classification and prognosis systems for periimplantitis have been proposed: One classification is based on etiology of periimplantitis and is divided into five categories. However, it fails to differentiate between mild, moderate, and advanced disease and does not consider that two or more etiologic factors may be present simultaneously and contributing to peri-implantitis on a given implant (16).

A more recent system classifies peri-implant status following surgical treatment (17). This is valuable in establishing a post treatment diagnosis and aids in postsurgical decision making but does not help the clinician in determining the method to use in treating early, moderate, or advanced peri-implantitis disease.

A more complicated classification system published in 2018 based on a literature research from 1967-2017 includes 6 Diagnostic Categories (DC-1-6) (18). For advanced disease it also includes that implant mobility, pain, positive BOP and/or suppuration can be present. Implant mobility in any degree of peri-implantitis indicate explantation as treatment. Thus, by including mobility in this classification for advanced peri-implantitis, treatment obviously becomes implant removal.

A more useful classification system is based on BOP, (and or suppuration), probing depth and degree of bone loss relative to the implant length (19). This system divides the disease into three classifications (Figure 1). When the clinical signs of inflammation are present, bone loss is then determined on a periapical radiograph comparing it to the $\%$ of the implant length. The value of this system is that it is simple to determine and is therefore used in a number of decision trees and prognosis systems for the treatment of periimplantitis. This is particularly valuable to the clinician in helping him/her to determine the treatment options, particularly if the implant has advanced periimplantitis.

A decision tree on the treatment of peri-implantitis published in 2011 was based on bone loss. For advanced peri-implantitis (when bone loss exceeds $1 / 2$ the implant length), the authors recommend that the implant be removed, the site redeveloped, and another implant be placed (20).

A prognosis system for peri-implant diseases (2015), based on a literature search of 101 articles, states that this system, "correctly predicted the likely outcome of peri implant disease up to 1 year post treatment for all examined implants" (21). They too recommend extraction and site development if a non-mobile implant has advanced peri-implantitis (bone loss $>1 / 2$ implant length). A more recent Decision Tree (2018) based on peri-implant defect morphology stated that "implant removal is advised when severe $(>50 \%)$ signs of radiographic MBL (marginal bone loss) are observed" (22). Thus, the recommendation in all of the above for the treatment of advanced peri-implantitis, is implant removal and site development. It is therefore not surprising that peri-implantitis is the main reason for late implant failure (81.9\%) (23).

Most late failing implantitis, due to peri-implantitis are not mobile, remain partially osseointegrated and may present a challenge for removal. Therefore, explanation may be invasive and neighboring teeth, bone and vital anatomical structures can be potentially harmed (24). The least invasive technique for explanation is to unscrew 
the implant by breaking the bone to implant interface. Commercial implant removal kits are available. However, when these are unable to reverse torque out the implant more invasive instruments have to be used. Trephining is the most invasive option for implant removal and should only be used when all other methods are exhausted (24).

Another method of implant removal uses laser therapy. A comparison between an Er,Cr:YSGG-laser and trephine approach for explanation, in an in vitro study on human mandibles on 12 implants, showed the laser to be less invasive but its use was more time consuming (25).

Depending on the extent of bone loss following removal of an implant with advanced peri-implantitis and considering bone loss of $>50 \%$ of the implant length is present, horizontal or vertical defects usually result after implant removal. Vertical and/or horizontal augmentation of the ridge is usually required. (Description of these are beyond the scope of this paper). However, two studies on survival/success rate of implant replacements for failed implants show survival rates of $71 \%$ and $83.5 \%$ respectively $(26,27)$. Studies by the same authors show survival rates of a third attempt to be $50 \%$ and $60 \%$ respectively $(26,28)$.

Therefore, successful treatment (and retention) of an implant with advanced peri-implantitis (bone loss $>50 \%$ of implant length) is obviously more desirable than explanation from a patient as well as clinicians' perspective in that it is less invasive, less costly, has less morbidity, and avoids a situation where post explanation the site would not be amenable for a replacement implant without additional ridge augmentation surgery.

\section{Peri-implantitis treatment}

Many surgical protocols have been proposed to treat periimplantitis of early to moderate severity (29). However, in a 5 -year follow-up study the surgical and antimicrobial strategy followed was successful only $58 \%$ of the time (30). In a retrospective longitudinal study of the treatment of 382 implants with peri-implantitis in 150 patients in a periodontal clinic, flap surgery with osteoplasty was the most common type of therapy (47\%) while regenerative surgery with bone substitute materials was the treatment in $20 \%$ of the cases. Overall, the mean success at patient level was $69 \%(31)$.

In fact, an excellent systematic review on the surgical management of peri-implantitis concluded that "the available evidence does not allow specific recommendations for the therapy of peri-implantitis" (32).
The treatment of implants with advanced peri-implantitis ( $>50 \%$ bone loss) presents even more of a challenge because most decision trees and prognosis systems indicate these implants should be removed. Moreover, treatment requires regenerative surgical procedures, and long-term data on success rates for regenerative surgical procedures is lacking. In fact, a systematic review and meta-analysis of the clinical outcome of regenerative approaches concluded that "there is limited evidence in the literature reporting long-term results (at least 36 months follow up) of the regenerative treatment of peri-implantitis" (33).

\section{Combined treatment resection/regeneration}

A number of researchers have based their surgical approach on the type of defect present: horizontal, vertical or a combination of the two (34). Combined surgical therapy of advanced peri-implantitis with 4-year followup was evaluated comparing two methods of surface decontaminations (SDCs) (35). Treatment of the supra and intrabony defects consisted of access flap surgery, granulation tissue removal, implantoplasty at buccal and supracrestal exposed implant parts, and implant SDC of the unmodified surfaces with (I) Er:YAG laser (ERL) or (II) plastic curets and cotton pellets and sterile saline (CPS). Both groups received augmentation with a natural bone mineral covered by a collagen membrane. At 48 months post treatment CPS-treated sites revealed higher reductions in mean BOP (CPS $85.2 \% \pm 16.4 \%)$ than ERL $(71.6 \% \pm 24.9 \%)$ and better clinical attachment level (CAL) values with CPS $(1.5 \pm 2.0 \mathrm{~mm})$ than with ERL $(1.2 \pm 2.0 \mathrm{~mm})$. In both groups clinical outcomes were not directly influenced by initial defect configuration or method of SDC. Moreover, in this study no data was given on bone gain or marginal recession.

However, a case series of 13 implants in 10 patients treated with a combination protocol was later published citing mucosal recession (MR) as a common finding with the combined treatment (36). The 13 implants in this study all had both supra and intrabony defects which underwent combined therapy. This again consisted of access flap surgery, implantoplasty at buccally and supracrestally exposed implant parts, and augmenting of the intrabony components with a natural bone mineral covered with native collagen membrane after SDC using subepithelial connective tissue curets and cotton pellets soaked in sterile saline. The one additive step was obtaining a (SECT) graft from the palate which intentionally was $50 \%$ wider than the mesiodistal defect 
and adapting it over the collagen membrane on the buccal aspect of the implants. Flaps were then repositioned to cover the SECT graft. Following a strict maintenance program at 6 months post-surgery. BOP was reduced $74.39 \% \pm 28.52 \%, P D$ was reduced $2.53 \pm 1.80 \mathrm{~mm}$, and CAL gain was $2.07 \pm 1.93 \mathrm{~mm}$.

Instead of MR there was an average gain in the level of marginal mucosa of $+0.07 \pm 0.5 \mathrm{~mm}$. Again, no measurements were performed for radiographic bone fill of the defects. The goal of using the SECT graft was to minimize buccal recession which usually follows use of the combined therapy protocol. In this case series the "gain" in marginal mucosa levels of $0.07 \mathrm{~mm}$ showed that this can be accomplished when a SECT graft is part of the protocol.

Another treatment decision tree recommended implant explanation when bone loss $\geq$ two thirds of implant length (37). For vertical and combined defects, the authors recommended removing the prosthesis and submerging the implant with the flap following GBR treatment. Using this decision tree in a 2-to-21-year retrospective evaluation by the same authors 57 implants in 45 patients were treated and followed [average follow up time 6:9 years (range, 2-27 years)] (38). A success rate of $70.2 \%$ was documented on the total 40 implants that were successfully treated, recurrence of periimplantitis occurred on 9 implants, and 8 implants were removed. However, the authors were treating moderate to advanced peri-implantitis. Except for implant SDC the authors used the same flap design, grafts, membrane barriers and suturing techniques used in their studies on vertical ridge augmentation (39).

\section{Rationale for the regenerative approach}

In most studies on the treatment of advanced periimplantitis the authors use "regenerative" protocols. The radiographic bone gain (RBG) obtained is measured by comparing pretreatment radiographs with the final follow up radiographs. Although this may measure "new bone", it, of course, does not denote regeneration or reosteointegration of bone to a previously denuded implant surface due to peri-implantitis. The proof for regeneration (re-osseointegration) requires histology of an implant and surrounding bone, which is difficult to obtain, (especially in the United States due to IRBA and ethical considerations), on an implant where successful bone fill and pocket reduction were achieved. However, two animal studies and a literature review of animal studies have demonstrated reosteointegration to a diseased implant surface following implant SDC and GBR techniques (40-42).

One human histological study in which regenerative treatment was used to treat an implant with periimplantitis did show histological regeneration (re-osseointegration) to an implant surface from which bone was lost to the disease (43). A second human histological autopsy study was performed 20 months after a regenerative surgery using a synthetic bone substitute to treat peri-implantitis. Reosteointegration was histologically observed (44). These can be considered "proof of principal" studies showing that regeneration of new bone to an implant surface denuded of bone due to periimplantitis is possible.

However, because of the limitations cited above, radiographic evidence and bone sounding are the parameters used in most studies of regenerative treatment of a periimplantitis to measure the amount of new bone formation.

"Regenerated" bone has also been demonstrated in several studies which used reentry surgery to view the bone and compare it to the initial surgical levels. One such study, using both submerged and non-submerged surgical approaches, to treat 9 implants in 6 female patients performed reentry procedures to compare direct visual evidence of defect fill for clinical measurements (45). Five of the six patients were treated with a submerged approach where the restoration was removed and the treated implant, bone and graft were submerged under the flap during the healing phase. The authors stated the peri implant bone debridement, implant SDC and detoxification were identical to those described in a previous article (37). All defects received composite, allografts combined with autogenous bone and covered with either an alloderm graft or ePTFE membrane. Reentry surgery was performed at either 12 or 22-month post-surgery, for abutment placement or for periodontal restorative treatment of an adjacent tooth, respectively. The intraoperative bone levels (as viewed at reentry) showed a mean bone fill of $91.3 \%$ ranging from $50 \%$ to $100 \%$. The mean pretreatment defect depth was $5.44 \mathrm{~mm}$. The mean post treatment defect depth was $0.44 \mathrm{~mm}$ with a mean bone gain of $4.88 \mathrm{~mm}$. A similar study following a regenerative surgical protocol on 12 periimplantitis effected implants in 5 patients had reentries performed 6-96 months post treatment (46). The treatment protocol differed from the previous reentry study and will be described in detail later in this chapter. Six of the 12 implants had advanced periimplantitis (defect depth 9-12 mm) and 6 implants had moderate periimplantitis (defect depth $3-5 \mathrm{~mm})$. Bone gain ranged from $2-9 \mathrm{~mm}(40-100 \%)$. No lesion in this case series either lost bone or registered "no" 
fill after treatment. In no case was the treatment surgery performed with a submerged protocol. No restoration was removed during the regenerative surgical procedures.

When looking at all surgical approaches to treat peri-implantitis a recent literature review, which was "a comprehensive overview of systematic reviews" concluded that "there was no strong evidence to suggest the most effective treatment intervention for peri-implantitis" (47). However, a narrative review of the literature of successful surgical protocols identified studies reporting positive clinical outcomes at 12 months or more of follow up (48). The later review concluded that heterogeneity of the studies made it impossible to determine a correlation between clinical outcome and SDC methods. However, it noted that most studies over 12 months reporting better treatment outcomes employed a bone replacement graft".

A number of case series studies using various protocols have been published with the goal of obtaining bone "regeneration" around peri-implantitis affected implants (49-52). The first of these studies used autogenous bone to augment open crater-formed peri-implant defects. However, it did not include implants with advanced peri-implantitis since all defects treated had bone loss (" $50 \%$ of the implant length") (49). The second study used a single intervention treating 36 implants in 22 patients. Defects were filled with autogenous bone mixed 1:1 with a xenogenic bone graft. Radiographic evaluation of the bone defects reveled a mean reduction of $3.5 \mathrm{~mm}$. However, the results were only 1 year post treatment and there was no mention of how many of the implants treated had advanced peri-implantitis (50). The third study included 20 patients, but all had moderate peri-implantitis (51). The fourth of these studies looked at the stability of bone regeneration over 3 years and reported a mean defect fill using a bone substitute alone of $1.3 \mathrm{~mm}$ and $1.6 \mathrm{~mm}$ when a resorbable membrane was used to cover the bone substitute. Again, no implants were diagnosed persurgically having advanced peri-implantitis (52).

A comparison of surgical protocols to treat advanced peri-implantitis is not possible because (I) implants where bone loss exceeds $50 \%$ of implant length are explanted and (II) not enough long-term date are available on studies which used 'regenerative" approach in treating implants with advanced peri-implantitis (33).

\section{8-step regenerative approach}

A study using a regenerative approach for the successful treatment of peri-implantitis reported very promising results (53). Of a total of 170 consecutively treated implants in 100 patients with 2-10-year follow-up (average followup time 3.6 years), only 2 implants were lost resulting in a $98.8 \%$ survival rate. BOP was eliminated in $91 \%$ of the treated implants. Probing depth reduction averaged $5.10 \mathrm{~mm}$, gain in bone level (determined by radiographs) averaged $1.77 \mathrm{~mm}$, and soft tissue marginal gain average $0.52 \mathrm{~mm}$. These outcomes were obtained with one surgical procedure on 140 implants, with two procedures on 18 implants, and with three procedures on 10 implants. Of the 170 implants treated 48 had advanced peri-implantitis ( $>50 \%$ bone loss) with $47 / 48(97.9 \%)$ of these implants having successful outcomes and were retained. The 8-step surgical protocol used was described in an earlier paper and begins with case selection (54) (Figure 2).

\section{Technique}

Case selection is primary for achieving successful outcomes. A thorough medical and dental history are taken. Smoking, a history of periodontitis, lack of compliance and poor oral hygiene, systematic disease (poorly controlled diabetes, cardiovascular disease, immunosuppression), lack of keratinized tissue and a history of one or more failure of implants are all considered high-risk factors for obtaining successful outcomes (14). With the exception of past implant failures all other risk factors should be addressed (and controlled) whenever possible prior to treatment of a periimplantitis affected implant. A previously cited narrative review of successful surgical protocols showed treatment of periodontitis in patients prior to periimplantitis treatment resulted in better treatment outcomes (48).

Implant mobility, poor implant position (excessive buccal or lingual placement, implants placed $<2 \mathrm{~mm}$ from an adjacent tooth or $<3 \mathrm{~mm}$ from an adjacent implant) result in an inability to access the implant surface for complete decontamination, are contraindications for treatment of advanced peri-implantitis with this regenerative protocol and are instead indications for explantation (Figure $3 A-3 D$ ).

\section{Flap access}

The second and third steps of this protocol include flap access and SDC. Prior to flap access all patients were premedicated with oral administration of amoxicillin 2,000 $\mathrm{mg}$ (Novo Pharma) or if the patients were allergic to amoxicillin, Clindamycin $600 \mathrm{mg}$ (Ohm Laboratories). Patients were continued on amoxicillin $500 \mathrm{mg}$ tid or 


\begin{tabular}{|lr|}
\hline \multicolumn{2}{|l|}{ Peri-implantitis treatment: 8 essential factors for success } \\
\hline 1) Case selection & Ensure adequate blood supply \\
\hline 2) Flap access & Emdogain and/or PDGF on implant surface \\
\hline 3) Surface decontamination & MFDBA and/or anorganic bovine bone + biologic (rhPDGF) \\
\hline 4) Defect debridement & (I) Adequate band of keratinized tissue: absorbable membrane \\
\hline 5) Defect fill & (II) Inadequate band of keratinized tissue: subepithelial connective \\
\hline 6) Coverage & tissue graft (submerged under the flap) \\
\hline 7) Coronal positioning of the flap & Complete coverage of membrane/graft \\
\hline 8) Professional maintenance/monitoring & Excellent homecare \\
\hline
\end{tabular}

Figure 2 Peri-implantitis treatment. Eight essential factors for success. PDGF, platelet derived growth factor; MFDBA, mineralized freezedried bone allograft; rhPDGF, recombinant platelet derived growth factor.

clindamycin $150 \mathrm{mg}$ qid for an additional 10 days. In a study of success of preoperative antibiotics on the placement of 2,641 implants, the results showed significantly fewer failures when preoperative antibiotics were used (55). Another literature review and meta-analysis of 4 randomized clinical trials (RCTs) showed a statistically significantly higher number of patients experiencing implant failures in the group not receiving antibiotics (56). Therefore, antibiotics were routinely given in treatment of advanced peri implant with the 8 -step regenerative protocol. The flap should extend at least one tooth anterior and one tooth posterior to the effected implant. The flap should be reflected to enable full access to the effected implant surface. However, the periosteum should be retained on the buccal and lingual bony surfaces beyond the area of required for SDC. This can be accomplished with periosteal releasing incisions, or where necessary, vertical incisions. The former preserves the blood supply of the bone around the implant and allows the flaps to be coronally positioned.

\section{SDC}

Following mechanical debridement, SDC is performed in conjunction with thorough debridement of the osseous defects and implant surfaces and includes the use of special graphite curettes (Gracey 13/14 curette) titanium curettes or titanium (204SD, Salvin Dental) and titanium brushes to remove retained cement and calculus. The osseous defect is debrided, and small decortications are made in the intrabony part of the defect with the tips of the Gracey curettes.

SDC using this protocol consists of a 7-step mechanical and chemical protocol and includes:

(I) The implant surface is treated with an air powder abrasive spray (prophy-Jet Dentsply) with a special contra angle tip; to reach all the areas using glycine for 60 seconds followed by:

(II) Saline spray alone with an irrigation device (Infinity irrigator, Ace surgical) for 1 minute. In a literature review of 27 articles that utilized the air powder abrasive, the authors concluded that the cleaning efficacy evaluated by removal of bacterial endotoxin ranged from $84 \%$ to $98 \%$ and removal of the bacterial biofilm was up to $100 \%$ according to the in-vitro studies (57). A warning about using the air powder abrasive which can leave particles on the implant surface and may result in subcutaneous emphysema was stated in an overview of surface detoxification and a case report on detoxification of implant surfaces $(58,59)$. However, the authors of the 8 -step regenerative protocol have found that a 1-minute spray with sterile saline with the air powder abrasive (without any powder) avoids adhesion of glycine particles to the implant surface. Moreover, when the tip of the instrument is used at a $45^{\circ}$ angle to the implant subcutaneous emphysema were avoided (60). The authors of the 8 -step protocol also recommend that the flaps be held down firmly against bone with a gauze soaked in saline and held with a mouth mirror to prevent 

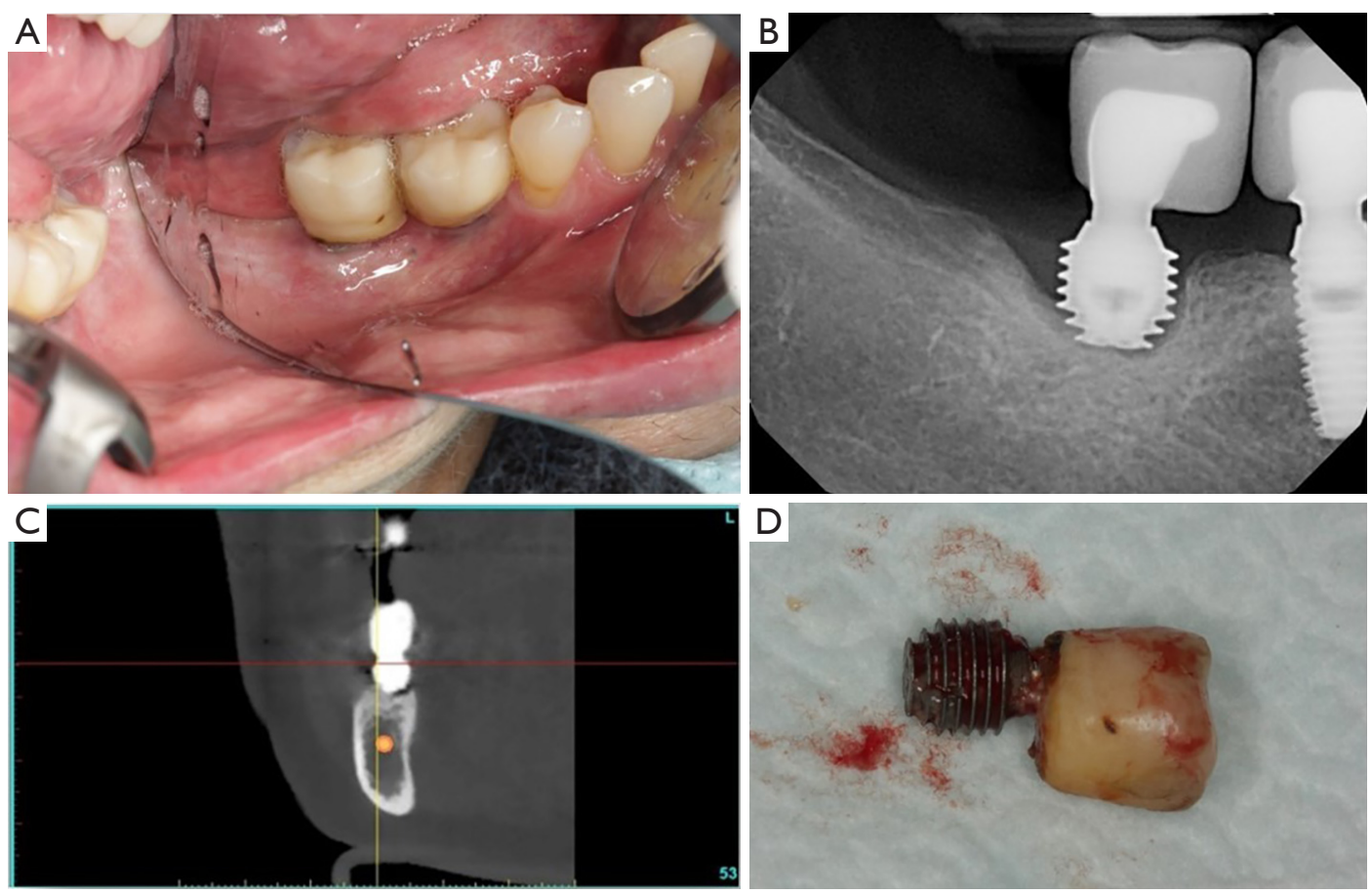

Figure 3 Advanced peri-implantitis around a mandibular right second molar which according to case selection criteria was determined to be hopeless because the implant was mobile due to bone loss down to and around implants apex. (A) Clinical photo of hopeless mandibular right first molar implant with advanced peri-implantitis. Patient wished to have surgery to save the implant. (B) Radiograph of implant showing bone loss around entire mobile implant. (C) Cone beam computed tomography scan of implant with bone missing to apex. (D) Implant removed shows microbiome to apex.

any powder or spray from going under the flap (Figure 4).

(III) Application of tetracycline $50 \mathrm{mg}(\mathrm{mL})$ with nonwoven gauze.

(IV) This is followed by a second use of the air powered abrasive with glycine for 60 seconds and then rinsed for 60 seconds with sterile saline.

(V) Application of $0.12 \%$ chlorhexidine gluconate (Peridex oral rinse 3M ESPE) applied to the implant surface with non-woven gauze for 30 seconds.

(VI) Sixty seconds of re-irrigation with sterile saline.

(VII) The implant surface is then treated with citric acid $(\mathrm{pH}=1)$ in a $50 \%$ saturated solution applied with nonwoven gauze for 30 seconds to one minute. This is followed by vigorous rinsing with sterile water for one to two minutes. A literature review concluded that citric acid "demonstrated the greatest efficacy in cleaning the contaminated surface", as evidenced by scanning electron microscopy (61). Moreover, a research article which used guinea pigs showed that citric acid $(50 \% \mathrm{pH}-1)$ when applied for only 30 seconds to bone enhanced adhesion and spreading of pre osteoblasts (62).

In a comprehensive review of the literature on implant surface detoxification it was stated that "at this time all techniques/agents have been shown to be equally effective to detoxify the contaminated implant surface". The authors also concluded that "comparative studies of different detoxification methods were heterogeneous leaving a few voids when selecting a specific technique" (63). Moreover, in the consensus report of the Sixth European Workshop on Periodontology the authors stated that "no single method of SDC (chemical agents, air abrasives and lasers) was found to be superior" (12). However, the combined technique used in the 8-step regenerative approach using mechanical as well as chemical detoxification of the implant was tested on 14 hopeless implants in a comparative study. The same algorithm used in the 8 steps regenerative study was tested on 6 implants versus a 6-implant untreated controls vs. 2 implants mechanically treated and followed by rubbing the 


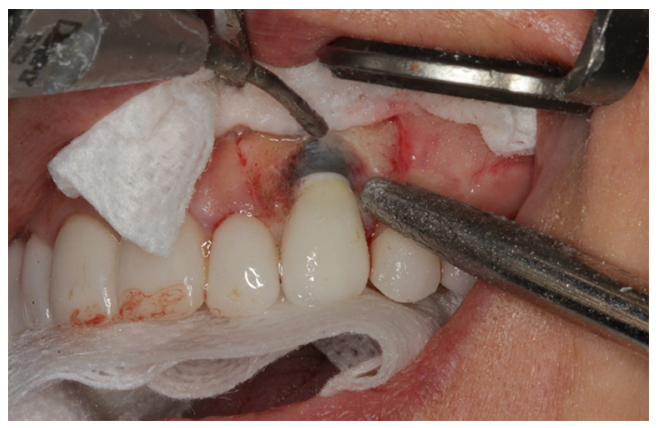

Figure 4 Air powder abrasive used with tip at a 45-degree angle, and flap held down with saline soaked gauze and mouth mirror to prevent air emphysema.

surface with sterile saline. Following treatment all implants were placed in culture with human osteoprogenitor cells for 72 hours and evaluated with a scanning electron microscope. The six test implants all demonstrated robust attachment and proliferation of the normal human osteoprogenitor cells on their prior exposed and then decontaminated surfaces. All of the untreated controls and mechanically debrided, sterile water treated implants, failed to demonstrate the same success (64).

An often-recommended method of surface detoxification uses lasers. A systemic review of the efficacy of different types of lasers concluded that the ERL exhibits strong bactericidal effects against periodontopathic bacteria while the $\mathrm{CO}_{2}$ laser was reported to be safe and able to enhance bone regeneration. The authors also state that no human studies evaluated the effect of the Nd:YAG laser on periimplantitis and because of the paucity of studies "no firm conclusion can be drawn "regarding the use of lasers to treat peri-implantitis" (65).

However, a recent study used the ERL for SDC followed by bone grafting (66). Although various type of lasers has been used to treat peri-implantitis, a caveat should be included based on this and other studies. The author used the ERL with "2 complete passes" to perform surface detoxification or until surface appearance changes were seen on the implant surface (with a change in reflective quality of the implant surface or dark gray discoloration) as suggested in a study (67). Although there have been a number of excellent studies on use of the ERL to decontaminate an infected implant surface, the authors of these studies had significant experience prior to the study $(68,69)$. Clinicians with less experience should be extremely careful when using any of the lasers to treat peri-implantitis. Over 60 patients have been referred to the authors private practice with necrotic and overheated bone due to incorrect and improper use of lasers to treat peri-implantitis. The laser may be a valuable adjunct in the treatment of advanced peri-implantitis, but training and experience are necessary and essential to avoid severe complications.

The 8-step regenerative approached used no lasers for SDC following SDC with mechanical means, the air powder abrasive, citric acid, and saline.

\section{Biologics and bone grafts}

Enamel matrix derivative and/or recombinant platelet derived growth factor (rhPDGF) are then applied to the cleaned implant surface. One invitro study concluded that the combination of EMD and PDGF-BD produces greater proliferation and wound fill effects on periodontal ligament sells (PDL) cells than each by themselves (70). The defect is then filled, both the intrabony and suprabony parts with a bone graft of MFDBA + anorganic bovine bone (3/1 ratio) combined with rhPDGF (71-73).

\section{Membrane barriers-collagen vs. connective tissue graft}

When treating implants that have adequate band of keratinized tissue $\geq 2 \mathrm{~mm}$, a resorbable collagen barrier is shaped and used and shaped to cover the buccal and interproximal bone graft material. Another piece of barrier is fitted to cover the lingual bone graft. Barriers are sutured or tacked down with surgical tacks (Figure 5).

In the treatment of advanced peri-implantitis it had been noted that there is a strong risk of extreme implant exposure that results in a loss of soft tissue morphology and keratinized tissue (74). This is the reason that use of the combined therapy for treatment of advance peri-implantitis includes a SECT graft. In the 8-step regenerative protocol, on implants that have an inadequate band of keratinized tissue, ( $<2 \mathrm{~mm}$ of keratinized mucosa), a SECT graft is harvested from the palate and shaped to cover the buccal and interproximal bone (75). The SECT is sutured in place with 4-0 Vicryl sutures. The importance of keratinized mucosa was discussed in a systematic review (76). The conclusion of this review was that the lack of inadequate $\mathrm{KM}$ around endosseous dental implants "is associated with more plaque accumulation, tissue inflammation, $M R$ and attachment loss (AL)". A prospective longitudinal comparison study of 3 surgical therapies for peri-implantitis concluded 
at the 3-year follow-up that the treatment group that used SECT grafts maintained the greatest $\mathrm{KM}$ width (77). The addition of the graft also reduces the amount of postoperative recession. Graft coverage is followed by coronally positioning of the flap. If the flap is unable to completely cover the graft (or if there is tension while covering the membrane and graft) additional periosteal incisions or extension of the vertical incisions are indicated to enable complete coverage of the membrane, graft, and implant surface without tension. If a provisional is being placed, it should be adjusted so there is no pressure on the flap.

\section{Maintenance}

The last of the 8 steps in the regenerative protocol is professional maintenance combined with excellent homecare. In a 5-year follow-up study of maintenance therapy in patients who maintained a high level of oral hygiene following the surgical treatment of peri-implantitis and monitored every 6 months it was found that conditions obtained following peri-implantitis surgery were maintained stable (78). Another 5-year study of patients with preexisting peri mucositis showed that without preventive maintenance $43.9 \%$ developed peri-implantitis vs. $18.0 \%$ with preventive maintenance (79). In a commentary "Routine Maintenance of Dental Implants" was recommended by the authors along the guidelines for patients with periodontal disease. This is certainly necessary for any implant following successful treatment of peri-implantitis (80). Patients in the 8 -step regenerative study were seen every 3 months for maintenance and monitoring (53). Care was taken to clean the implant and review homecare at each maintenance visit. In a literature review of 47 articles on implant maintenance the authors concluded that maintenance and monitoring involving the assessment of the patients general and oral health, profession implant maintenance (they suggest every 3 months) and diligent patient home care "are" critical factors that will ensure long-term success of implants (81). This is even more important following treatment of an implant with advanced peri-implantitis to avoid disease recurrence and loss of the soft and hard tissue achieved with successful regenerative therapy.

Retention of an implant with advanced peri-implantitis with the 8-step regenerative surgical protocol, as mentioned earlier is certainly cost effective. A published costeffectiveness analysis agreed with this statement and stressed supportive implant therapy (SIT) to prevent and identify recurrence of the disease (82). This again is key to maintaining the successful outcomes obtained with the 8 -step regenerative approach.

\section{Outcomes}

The 8-step regenerative protocol used to treat advanced peri-implantitis, when successful, avoids the necessity to remove the diseased implant and the time, morbidity, and expense of rebuilding the site and placing a new implant. It does not require removal of the restoration that the implant is supporting. Although it may require as many as 3 procedures, a successful outcome is usually welcomed by the patient and clinician. The protocol, as mentioned earlier, has a $98.8 \%$ successful outcome rate in the treatment of 170 implants (48 with advanced periimplantitis).

\section{Conclusions}

The keys for success of this 8-step protocol in the treatment of advanced peri-implantitis are:

(I) Proper case selection. High-risk patients, where the risk factors cannot be eliminated, are not candidates for this protocol. Implants that are mobile should be explanted. In addition, severely malposed implants are not candidates.

(II) Obtaining complete access for implant decontamination.

(III) Use of a bone graft and biologic with a collagen barrier when there is enough $(>2 \mathrm{~mm})$ keratinized tissue or a SECT graft as a barrier if there is inadequate keratinized tissue.

(IV) Proper maintenance-patients willing to return every 3 months for maintenance and monitoring and follow excellent home care procedure. If a patient is not compliant or refuses to follow the maintenance protocol, they are not candidates for this protocol.

(V) Lastly, the surgery should be performed by an experienced clinician who is familiar with bone and soft tissue grafting.

The 8 -step regenerative protocol has been shown to be effective in treatment of advanced peri-implantitis and avoids the removal of an implant with this level of disease. More studies however are necessary to confirm the validity of the outcomes achieved in long-term studies using this 

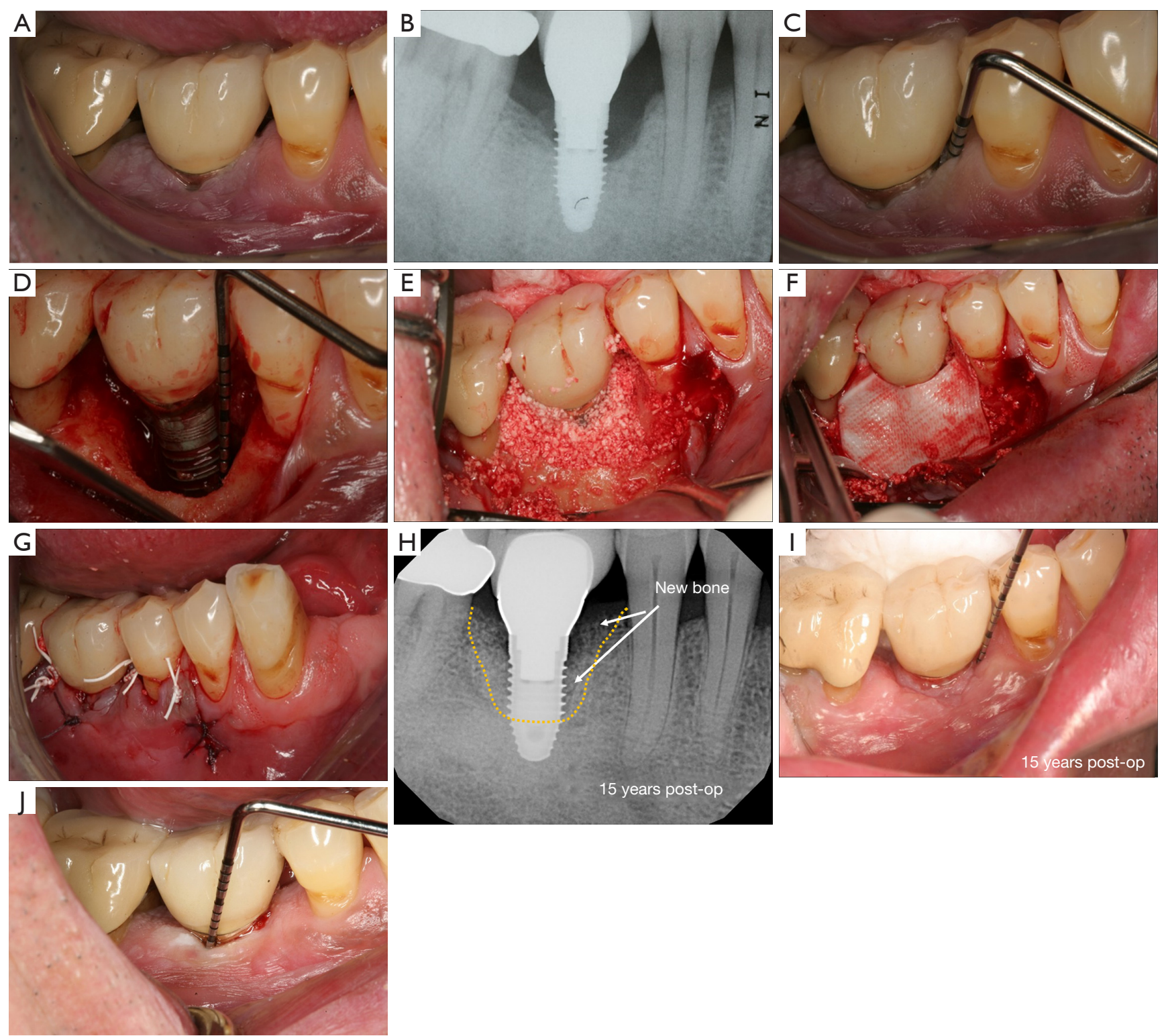

Figure 5 Treatment of advanced peri-implantitis on a mandibular right first molar from preop to 15 years postop. (A) Mandibular right first molar implant with BOP and advanced peri-implantitis. (B) Periapical radiograph. Appears bone loss is $50 \%$ of the implant surface. (C) $12 \mathrm{~mm}$ probing depth on mesial of implant. (D) Flap reflected shows bone loss of $11 \mathrm{~mm}$ from crown margin to bone defect indicating advanced peri-implantitis $>50 \%$ bone loss. Five mm of infrabony and 6 mm of suprabony defects. (E) Bone graft of mineralized freeze-dried bone and anorganic bone (3/1 ratio) mixed with rhPDGF fills infra + suprabony parts of the defects following surface decontamination and enamel matrix derivative + rhPDGF placed on decontaminated implant surface. (F) Contoured absorbable membrane covering buccal and interproximal bone. A second contoured membrane covered the lingual graft. (G) Flap coronally positioned to cover implant surface, graft, and membrane barriers. (H) 15 -year post op shows complete fill of new bone and outline of original defect. (I) 15 -year post op photo shows $1 \mathrm{~mm}$ probing depth on mesial aspect. (J) 15 -year "sounding" on the buccal under local anesthesia shows $3 \mathrm{~mm}$ bone depth. BOP, bleeding on probing. Published by permission of Wiley and Sons from an article: Froum SJ. Regenerative Treatment for a Peri-Implantitis-Affected Implant: A Case Report. Clin Adv Periodontics 2013;3:140-6. 
protocol.

\section{Acknowledgments}

Funding: None.

\section{Footnote}

Provenance and Peer Review: This article was commissioned by the Guest Editor (Ole T. Jensen) for the series "Current Advances in Treatment of Peri-Implantitis" published in Frontiers of Oral and Maxillofacial Medicine. The article has undergone external peer review.

Conflicts of Interest: The author has completed the ICMJE uniform disclosure form (available at https://fomm. amegroups.com/article/view/10.21037/fomm-21-30/ coif). The series "Current Advances in Treatment of PeriImplantitis" was commissioned by the editorial office without any funding or sponsorship. The author has no other conflicts of interest to declare.

Ethical Statement: The author is accountable for all aspects of the work in ensuring that questions related to the accuracy or integrity of any part of the work are appropriately investigated and resolved.

Open Access Statement: This is an Open Access article distributed in accordance with the Creative Commons Attribution-NonCommercial-NoDerivs 4.0 International License (CC BY-NC-ND 4.0), which permits the noncommercial replication and distribution of the article with the strict proviso that no changes or edits are made and the original work is properly cited (including links to both the formal publication through the relevant DOI and the license). See: https://creativecommons.org/licenses/by-nc-nd/4.0/.

\section{References}

1. Albrektsson T, Isdor F. Consensus report of session IV. In: Lang NP, Karring T. editors. Proceeding of the First European Workshop on Periodontology. London: Quintessence, 1994:365-9.

2. Caton JG, Armitage G, Berglundh T, et al. A new classification scheme for periodontal and peri-implant diseases and conditions - Introduction and key changes from the 1999 classification. J Periodontol 2018;89 Suppl 1:S1-8.
3. Schwarz F, Derks J, Monje A, et al. Peri-implantitis. J Periodontol 2018;89 Suppl 1:S267-90.

4. Mombelli A, van Oosten MA, Schurch E Jr, et al. The microbiota associated with successful or failing osseointegrated titanium implants. Oral Microbiol Immunol 1987;2:145-51.

5. Hultin M, Gustafsson A, Hallström H, et al. Microbiological findings and host response in patients with peri-implantitis. Clin Oral Implants Res 2002;13:349-58.

6. Subramani K, Jung RE, Molenberg A, et al. Biofilm on dental implants: a review of the literature. Int J Oral Maxillofac Implants 2009;24:616-26.

7. Charalampakis G, Leonhardt $\AA$, Rabe P, et al. Clinical and microbiological characteristics of peri-implantitis cases: a retrospective multicentre study. Clin Oral Implants Res 2012;23:1045-54.

8. Oates TW. THEMATIC ABSTRACT REVIEW: Understanding Peri-implantitis Through the Lens of the Oral Microbiome. Int J Oral Maxillofac Implants 2015;30:503-6.

9. Derks J, Tomasi C. Peri-implant health and disease. A systematic review of current epidemiology. J Clin Periodontol 2015;42 Suppl 16:S158-71.

10. Roos-Jansåker AM, Renvert H, Lindahl C, et al. Surgical treatment of peri-implantitis using a bone substitute with or without a resorbable membrane: a prospective cohort study. J Clin Periodontol 2007;34:625-32.

11. Behiens E, Kolenda I, Terheydoen H, et al. Langzeitergebnisse des ITI-Implantatsystems. Implantologie 2004;2:133-47.

12. Lindhe J, Meyle J; Group D of European Workshop on Periodontology. Peri-implant diseases: Consensus Report of the Sixth European Workshop on Periodontology. J Clin Periodontol 2008;35:282-5.

13. Tomasi C, Derks J. Clinical research of peri-implant diseases--quality of reporting, case definitions and methods to study incidence, prevalence and risk factors of peri-implant diseases. J Clin Periodontol 2012;39 Suppl 12:207-23.

14. Smeets R, Henningsen A, Jung O, et al. Definition, etiology, prevention and treatment of peri-implantitis--a review. Head Face Med 2014;10:34.

15. Figuero E, Graziani F, Sanz I, et al. Management of periimplant mucositis and peri-implantitis. Periodontol 2000 2014;66:255-73.

16. Sarmiento HL, Norton MR, Fiorellini JP. A Classification System for Peri-implant Diseases and Conditions. Int J Periodontics Restorative Dent 2016;36:699-705. 
17. Ravidà A, Galli M, Siqueira R, et al. Diagnosis of periimplant status after peri-implantitis surgical treatment: Proposal of a new classification. J Periodontol 2020;91:1553-61.

18. Tallarico M, Canullo L, Wang HL, et al. Classification Systems for Peri-implantitis: A Narrative Review with a Proposal of a New Evidence-Based Etiology Codification. Int J Oral Maxillofac Implants 2018;33:871-9.

19. Froum SJ, Rosen PS. A proposed classification for peri-implantitis. Int J Periodontics Restorative Dent 2012;32:533-40.

20. Okayasu K, Wang HL. Decision tree for the management of periimplant diseases. Implant Dent 2011;20:256-61.

21. Decker AM, Sheridan R, Lin GH, et al. A Prognosis System for Periimplant Diseases. Implant Dent 2015;24:416-21.

22. Sinjab K, Garaicoa-Pazmino C, Wang HL. Decision Making for Management of Periimplant Diseases. Implant Dent 2018;27:276-81.

23. Anitua E, Piñas L, Begoña L, et al. Prognosis of Dental Implants Immediately Placed in Sockets Affected by Periimplantitis: A Retrospective Pilot Study. Int J Periodontics Restorative Dent 2017;37:713-9.

24. Froum S, Yamanaka T, Cho SC, et al. Techniques to remove a failed integrated implant. Compend Contin Educ Dent 2011;32:22-32.

25. Hajji M, Franzen R, Grümer S, et al. Removal of Dental Implants Using the Erbium,Chromium:YttriumScandium-Gallium-Garnet Laser and the Conventional Trephine Bur: An in Vitro Comparative Study. Photomed Laser Surg 2016;34:61-7.

26. Grossmann Y, Levin L. Success and survival of single dental implants placed in sites of previously failed implants. J Periodontol 2007;78:1670-4.

27. Machtei EE, Mahler D, Oettinger-Barak O, et al. Dental implants placed in previously failed sites: survival rate and factors affecting the outcome. Clin Oral Implants Res 2008;19:259-64.

28. Machtei EE, Horwitz J, Mahler D, et al. Third attempt to place implants in sites where previous surgeries have failed. J Clin Periodontol 2011;38:195-8.

29. Heitz-Mayfield LJA, Salvi GE, Mombelli A, et al. Antiinfective surgical therapy of peri-implantitis. A 12-month prospective clinical study. Clin Oral Implants Res 2012;23:205-10.

30. Leonhardt A, Dahlén G, Renvert S. Five-year clinical, microbiological, and radiological outcome following treatment of peri-implantitis in man. J Periodontol 2003;74:1415-22.
31. Lagervall M, Jansson LE. Treatment outcome in patients with peri-implantitis in a periodontal clinic: a retrospective study. J Periodontol 2013;84:1365-73.

32. Heitz-Mayfield LJ, Mombelli A. The therapy of periimplantitis: a systematic review. Int J Oral Maxillofac Implants 2014;29 Suppl:325-45.

33. Khoshkam V, Suárez-López Del Amo F, Monje A, et al. Long-term Radiographic and Clinical Outcomes of Regenerative Approach for Treating Peri-implantitis: A Systematic Review and Meta-analysis. Int J Oral Maxillofac Implants 2016;31:1303-10.

34. Schwarz F, Sahm N, Schwarz K, et al. Impact of defect configuration on the clinical outcome following surgical regenerative therapy of peri-implantitis. J Clin Periodontol 2010;37:449-55.

35. Schwarz F, Hegewald A, John G, et al. Four-year follow-up of combined surgical therapy of advanced peri-implantitis evaluating two methods of surface decontamination. J Clin Periodontol 2013;40:962-7.

36. Schwarz F, Sahm N, Becker J. Combined surgical therapy of advanced peri-implantitis lesions with concomitant soft tissue volume augmentation. A case series. Clin Oral Implants Res 2014;25:132-6.

37. Parma-Benfenati S, Roncati M, Tinti C. Treatment of peri-implantitis: surgical therapeutic approaches based on peri-implantitis defects. Int J Periodontics Restorative Dent 2013;33:627-33.

38. Parma-Benfenati S, Tinti C, Romano F, et al. Long-Term Outcome of Surgical Regenerative Treatment of Periimplantitis: A 2- to 21-Year Retrospective Evaluation. Int J Periodontics Restorative Dent 2020;40:487-96.

39. Tinti C, Parma-Benfenati S. Vertical ridge augmentation: surgical protocol and retrospective evaluation of 48 consecutively inserted implants. Int J Periodontics Restorative Dent 1998;18:434-43.

40. Parlar A, Bosshardt DD, Cetiner D, et al. Effects of decontamination and implant surface characteristics on reosseointegration following treatment of peri-implantitis. Clin Oral Implants Res 2009;20:391-9.

41. Alhag M, Renvert S, Polyzois I, et al. Re-osseointegration on rough implant surfaces previously coated with bacterial biofilm: an experimental study in the dog. Clin Oral Implants Res 2008;19:182-7.

42. Renvert S, Polyzois I, Maguire R. Re-osseointegration on previously contaminated surfaces: a systematic review. Clin Oral Implants Res 2009;20 Suppl 4:216-27.

43. Fletcher P, Deluiz D, Tinoco EM, et al. Human Histologic Evidence of Reosseointegration Around an Implant 
Affected with Peri-implantitis Following Decontamination with Sterile Saline and Antiseptics: A Case History Report. Int J Periodontics Restorative Dent 2017;37:499-508.

44. Kim S, Hu KS, Jung UW. Reosseointegration After Regenerative Surgical Therapy Using a Synthetic Bone Substitute for Peri-implantitis: Human Autopsy Study. Int J Periodontics Restorative Dent 2018;38:585-91.

45. Parma-Benfenati S, Roncati M, Galletti P, et al. Periimplantitis Treatment with a Regenerative Approach: Clinical Outcomes on Reentry. Int J Periodontics Restorative Dent 2015;35:625-36.

46. Froum SJ, Rosen PS. Reentry evaluation following treatment of peri-implantitis with a regenerative approach. Int J Periodontics Restorative Dent 2014;34:47-59.

47. Ting M, Craig J, Balkin BE, et al. Peri-implantitis: A Comprehensive Overview of Systematic Reviews. J Oral Implantol 2018;44:225-47.

48. Froum SJ, Dagba AS, Shi Y, et al. Successful Surgical Protocols in the Treatment of Peri-Implantitis: A Narrative Review of the Literature. Implant Dent 2016;25:416-26.

49. Khoury F, Buchmann R. Surgical therapy of peri-implant disease: a 3-year follow-up study of cases treated with 3 different techniques of bone regeneration. J Periodontol 2001;72:1498-508.

50. Wiltfang J, Zernial O, Behrens E, et al. Regenerative treatment of peri-implantitis bone defects with a combination of autologous bone and a demineralized xenogenic bone graft: a series of 36 defects. Clin Implant Dent Relat Res 2012;14:421-7.

51. Schwarz F, Sahm N, Bieling K, et al. Surgical regenerative treatment of peri-implantitis lesions using a nanocrystalline hydroxyapatite or a natural bone mineral in combination with a collagen membrane: a four-year clinical follow-up report. J Clin Periodontol 2009;36:807-14.

52. Roos-Jansåker AM, Lindahl C, Persson GR, et al. Longterm stability of surgical bone regenerative procedures of peri-implantitis lesions in a prospective case-control study over 3 years. J Clin Periodontol 2011;38:590-7.

53. Froum SJ, Froum SH, Rosen PS. A Regenerative Approach to the Successful Treatment of Peri-implantitis: A Consecutive Series of 170 Implants in 100 Patients with 2- to 10-Year Follow-up. Int J Periodontics Restorative Dent 2015;35:857-63.

54. Froum SJ, Froum SH, Rosen PS. Successful management of peri-implantitis with a regenerative approach: a consecutive series of 51 treated implants with 3 - to 7.5-year follow-up. Int J Periodontics Restorative Dent 2012;32:11-20.
55. Dent CD, Olson JW, Farish SE, et al. The influence of preoperative antibiotics on success of endosseous implants up to and including stage II surgery: a study of 2,641 implants. J Oral Maxillofac Surg 1997;55:19-24.

56. Esposito M, Grusovin MG, Loli V, et al. Does antibiotic prophylaxis at implant placement decrease early implant failures? A Cochrane systematic review. Eur J Oral Implantol 2010;3:101-10.

57. Tastepe CS, van Waas R, Liu Y, et al. Air powder abrasive treatment as an implant surface cleaning method: a literature review. Int J Oral Maxillofac Implants 2012;27:1461-73

58. Valderrama P, Wilson Jr TG. Detoxification of implant surfaces affected by peri-implant disease: an overview of surgical methods. Int J Dent 2013;2013:740680.

59. Bergendal T, Forsgren L, Kvint S, et al. The effect of an airbrasive instrument on soft and hard tissues around osseointegrated implants. A case report. Swed Dent J 1990;14:219-23.

60. Augthun M, Tinschert J, Huber A. In vitro studies on the effect of cleaning methods on different implant surfaces. J Periodontol 1998;69:857-64.

61. Ntrouka VI, Slot DE, Louropoulou A, et al. The effect of chemotherapeutic agents on contaminated titanium surfaces: a systematic review. Clin Oral Implants Res 2011;22:681-90.

62. de Rezende ML, Coesta PT, de Oliveira RC, et al. Bone demineralization with citric acid enhances adhesion and spreading of preosteoblasts. J Periodontol 2015;86:146-54.

63. Suarez F, Monje A, Galindo-Moreno P, et al. Implant surface detoxification: a comprehensive review. Implant Dent 2013;22:465-73.

64. Rosen PS, Qari M, Froum SJ, et al. A Pilot Study on the Efficacy of a Treatment Algorithm to Detoxify Dental Implant Surfaces Affected by Peri-implantitis. Int J Periodontics Restorative Dent 2018;38:261-7.

65. Natto ZS, Aladmawy M, Levi PA Jr, et al. Comparison of the efficacy of different types of lasers for the treatment of peri-implantitis: a systematic review. Int J Oral Maxillofac Implants 2015;30:338-45.

66. Clem D, Gunsolley JC. Peri-implantitis Treatment Using Er:YAG Laser and Bone Grafting. A Prospective Consecutive Case Series Evaluation: 1 Year Posttherapy. Int J Periodontics Restorative Dent 2019;39:479-89.

67. Yamamoto A, Tanabe T. Treatment of peri-implantitis around TiUnite-surface implants using Er:YAG laser microexplosions. Int J Periodontics Restorative Dent 2013;33:21-30. 
68. Nevins M, Nevins ML, Yamamoto A, et al. Use of Er:YAG laser to decontaminate infected dental implant surface in preparation for reestablishment of bone-toimplant contact. Int J Periodontics Restorative Dent 2014;34:461-6.

69. Norton MR. Efficacy of Er:YAG Laser in the Decontamination of Peri-implant Disease: A One-Year Prospective Closed Cohort Study. Int J Periodontics Restorative Dent 2017;37:781-8.

70. Chong CH, Carnes DL, Moritz AJ, et al. Human periodontal fibroblast response to enamel matrix derivative, amelogenin, and platelet-derived growth factorBB. J Periodontol 2006;77:1242-52.

71. Lynch SE. Bone regeneration techniques in the orofacial region. In: Lieberman JR, Friedlaender GE. editors. Bone Regeneration and Repair. Cham: Springer nature, 2005: 359-390.

72. Friedlaender GE, Lin S, Solchaga LA, et al. The role of recombinant human platelet-derived growth factorBB (rhPDGF-BB) in orthopaedic bone repair and regeneration. Curr Pharm Des 2013;19:3384-90.

73. Nevins, M, Nevins ML, Simion M, et al. Recombinant platelet-derived growth factor (PDGF) in regenerative dentistry. In "Stem Cells and Regenerative Medicine". Eds. Marx RE, Miller RB. North Palm Beach: Best Publishing Co., 2020:167-82.

74. McCrea SJ. Advanced peri-implantitis cases with

\section{doi: $10.21037 /$ fomm-21-30}

Cite this article as: Froum SJ. Treatment of advanced peri-implantitis with regenerative therapy: a review of the rationale, technique and outcomes. Front Oral Maxillofac Med 2022;4:37. radical surgical treatment. J Periodontal Implant Sci 2014;44:39-47.

75. Langer B, Langer L. Subepithelial connective tissue graft technique for root coverage. J Periodontol 1985;56:715-20.

76. Lin GH, Chan HL, Wang HL. The significance of keratinized mucosa on implant health: a systematic review. J Periodontol 2013;84:1755-67.

77. Dalago HR, Perrotti V, Torres de Freitas SF, et al. Prospective longitudinal comparison study of surgical therapies for peri-implantitis: 3-year follow-up. Aust Dent J 2019;64:237-45.

78. Serino G, Turri A, Lang NP. Maintenance therapy in patients following the surgical treatment of periimplantitis: a 5-year follow-up study. Clin Oral Implants Res 2015;26:950-6.

79. Costa FO, Takenaka-Martinez S, Cota LO, et al. Periimplant disease in subjects with and without preventive maintenance: a 5-year follow-up. J Clin Periodontol 2012;39:173-81.

80. Wilson TG Jr, Valderrama P, Rodrigues DB. The case for routine maintenance of dental implants. J Periodontol 2014;85:657-60.

81. Gulati M, Govila V, Anand V, et al. Implant Maintenance: A Clinical Update. Int Sch Res Notices 2014;2014:908534.

82. Schwendicke F, Tu YK, Stolpe M. Preventing and Treating Peri-Implantitis: A Cost-Effectiveness Analysis. J Periodontol 2015;86:1020-9. 